\title{
Cerebellar and Frontal Hypometabolism in Alcoholic Cerebellar Degeneration Studied with Positron Emission Tomography
}

\author{
Sid Gilman, MD, ${ }^{*}$ Kenneth Adams, PhD, $\uparrow$ Robert A. Koeppe, PhD $\neq$ Stanley Berent, $\mathrm{PhD},{ }^{*} \dagger$ \\ Karen J. Kluin, MS, $\&$ Jack G. Modell, MD, $†$ Phillip Kroll, MD, $\dagger$ and James A. Brunberg, MD*ஈ
}

\begin{abstract}
Local cerebral metabolic rate for glucose was studied utilizing ${ }^{18} \mathrm{~F}$-2-fluoro-2-deoxy-D-glucose and positron emission tomography (PET) in 14 chronically alcohol-dependent patients and 8 normal control subjects of similar age and sex. Nine of the 14 patients (Group A) had clinical signs of alcoholic cerebellar degeneration, and the remaining 5 (Group B) did not have signs of alcoholic cerebellar degeneration. PET studies of Group A revealed significantly decreased local cerebral metabolic rates for glucose in the superior cerebellar vermis in comparison with the normal control subjects. Group B did not show decreased rates in the cerebellum. Both Groups A and B showed decreased local cerebral metabolic rates for glucose bilaterally in the medial frontal area of the cerebral cortex in comparison with the normal control subjects. The severity of the clinical neurological impairment was significantly correlated with the degree of hypometabolism in both the superior cerebellar vermis and the medial frontal region of the cerebral cortex. The degree of atrophy detected in computed tomography scans was significantly correlated with local cerebral metabolic rates in the medial frontal area of the cerebral cortex, but not in the cerebellum. The data indicate that hypometabolism in the superior cerebellar vermis closely follows clinical symptomatology in patients with alcoholic cerebellar degeneration, and does not occur in alcohol-dependent patients without clinical evidence of cerebellar dysfunction. Hypometabolism in the medial frontal region of the cerebral cortex is a prominent finding in alcoholdependent patients with or without alcoholic cerebellar degeneration.
\end{abstract}

Gilman S, Adams K, Koeppe RA, Berent S, Kluin KJ, Modell JG, Kroll P, Brunberg JA.
Cerebellar and frontal hypometabolism in alcoholic cerebellar degeneration studied
with positron emission tomography. Ann Neurol 1990;28:775-785

Alcoholic cerebellar degeneration (ACD) is a disorder of chronic alcohol-dependent patients characterized by ataxia of gait and incoordination of the lower extremities without prominent involvement of arm movements, speech, or eye movements [1-8]. The disease usually evolves slowly for weeks or months, but it may occur abruptly [2-9]. The symptoms may improve or even completely clear with cessation of alcohol intake and nutritional improvement [10]; however, many patients reach a plateau and have a permanent disturbance of stance and gait $[1-3]$. ACD often develops in association with Wernicke's disease, but the neuropathological changes in the cerebellum are similar whether or not the clinical and neuropathological features of Wernicke's disease are present $[2,3]$. ACD is thought to result from nutritional deficiency, particularly thiamine depletion $[2-4,9,11,12]$; however, a direct toxic effect of alcohol and its metabolites
$[5,9,13-19]$ and electrolyte disorders $[20]$ have also been implicated.

The neuropathological changes in ACD consist of degeneration of neurons in the anterior and superior portions of the cerebellar vermis, with extension into the anterior lobes and flocculi in severe cases [2-4, $9,21]$. Degenerative changes in the cerebellum of chronic alcohol-dependent patients may be asymptomatic; up to $27 \%$ of the brains of chronic alcoholdependent patients show evidence of cerebellar degeneration at autopsy, often without a history of clinical signs [9, 21, 22].

The diagnosis of $A C D$ is usually not difficult in a nutritionally deprived, chronic alcohol-dependent patient with a typical history and neurological findings. Anatomical imaging studies with computed tomography (CT) or magnetic resonance (MR) can be helpful by demonstrating atrophy of the anterior and superior
From the Departments of *Neurology, †Psychiatry, †Internal Medicine, sphysical Medicine and Rehabilitation, and Radiology, The University of Michigan, and the Veterans Affairs Medical Center, Ann Arbor, MI.

Received Jan 24, 1990, and in revised form Jun 14. Accepted for publication Jun 18, 1990.
Address correspondence to Dr Gilman, Department of Neurology, The University of Michigan, 1914 Taubman Center, 1500 E. Medical Center Drive, Ann Arbor, MI 48109-0316. 
ispects of the cerebellar vermis $[23-27]$. In some paients with the adult onset of cerebellar ataxia, howzver, the diagnosis may be difficult, particularly if alzohol intake is not extreme and the nutritional history $s$ equivocal. There are many causes of ataxia of gait and incoordination of leg movements in the adult [28, 29]. Consequently, the diagnostic evaluation of ataxia in the adult often requires extensive testing.

Positron emission tomography (PET) with ${ }^{18} \mathrm{~F}-2$ fuoro-2-deoxy-D-glucose $\left({ }^{18} \mathrm{~F}-\mathrm{FDG}\right)$ offers an opportunity to evaluate the functional activity of the central nervous system (CNS). Recently, PET was employed as a diagnostic test for the evaluation of adult-onset ataxia in patients suspected of having olivopontocerebellar atrophy (OPCA) [30-32]. These studies demonstrated hypometabolism in the cerebellar hemispheres, cerebellar vermis, and brainstem, with no abnormality elsewhere in the cerebral hemispheres [30]. The present study was initiated to determine whether hypometabolism in ACD would be seen in the anterior and superior aspects of the cerebellar vermis, cortesponding to the site of the major neuropathological changes $[2-4,9]$. This study was also designed to determine whether the degree of hypometabolism corresponded to the severity of the clinical neurological disorder and to the degree of cerebellar atrophy detected in anatomical imaging studies. Since a previous ${ }^{18} \mathrm{~F}$ FDG PET study of chronic alcohol-dependent patients without $A C D$ revealed hypometabolism in the medial aspects of the frontal lobe [33], we also studied metabolic tate in the frontal area of the cerebral cortex. The hippocampal region of both temporal lobes was included in our analysis because of the well-documented destructive effects of chronic alcohol abuse on hippocampal neurons and the association of neuronal loss in this region to the memory deficits observed in these patients [3, 34-36]. A preliminary report of parts of this study has been published [37].

\section{Materials and Methods}

Fourteen male patients in the Alcohol Treatment Unit and the Outpatient Clinics of the Ann Arbor Veterans Administration Medical Center (AAVAMC) were studied. Patients were admitted to this investigation if they had a history of a gait disorder occurring in the course of severe, chronic alcohol dependence. The patients were seen by a neurologist (S. G.), who obtained a history and conducted a physical and neurological examination.

All patients had a history of severe, chronic alcohol dependence, and all except one had a history of malnutrition (Tables 1 and 2). The patients were studied after an average duration of 45 days of monitored abstinence, and no patient was studied sooner than 27 days after detoxification. All patients were admitted to an inpatient unit for detoxification and provided with a standard nutritional intake, including multiple vitamin therapy. All patients were observed to be eating normally prior to the scan. A dietitian reviewed their diets and ensured that they were adequate. Blood and urine examinations within 7 days prior to the scan showed no evidence of ketosis, and serum glucose levels were within the normal range. During the period of study, the patients were taking no medications known to affect CNS function, except for disulfiram (Antabuse). The patients had no history of nonmedical use of psychoactive medications that reflect primary polydrug abuse or exceed the following criteria: (1) any recreational injection of any drug at any time in their lives; (2) more than six uses of marijuana in the year preceding testing; (3) more than 20 total occasions of hallucinogen use (including phencyclidine hydrochloride [PCP]); (4) more than five noninjectable uses of heroin or opium in the preceding year; (5) more than 100 pills, tablets, or capsules of synthetic narcotics in the preceding year; (6) more than 15 uses of nonpharmaceutical amphetamines or cocaine ever in their lives; (7) more than 90 uses of pharmaceutical amphetamines or methylphenidate in their lives; (8) more than 30 (nonprescription) uses of minor tranquilizers (e.g., diazepam, chlordrazepoxide) in the year preceding testing; (9) more than 60 uses of sedative hypnotics in the year prior to the study; and (10) other use of exotic chemical comforts evaluated in context. All patients studied met the criteria. Patients with neurological disorders of any kind other than those resulting from alcoholism were excluded. Patients with a history of a primary psychiatric disease were also excluded. The studies were approved by the Institutional Review Boards (IRB) of the AAVAMC and the University of Michigan Medical Center (UMMC), and informed consent was obtained from all subjects.

The diagnosis of ACD was made on the basis of the history, physical examination, and neurological examination and by laboratory tests and $\mathrm{CT}$ scans to exclude other diseases. None of the patients with ACD had disorders of sensory function adequate to cause ataxia of movements. The laboratory tests included complete blood counts, serum profiles of hepatic and renal function, serum levels of vitamin $B_{12}$ and folic acid, and studies of thyroid function.

Clinical neurological function was evaluated with conventional physical examinations documented with videotape recordings. A rating scale was devised extending from 0 (no disturbance) to +++ (severe disturbance) for oculomotor function, gait, coordinated movements of the upper limbs (the finger-nose-finger test and rapidly alternating movements of the hands), and coordinated movements of the lower limbs (the heel-knee-shin test and rapidly alternating movements of the feet). An overall clinical neurological score indicating the severity of the cerebellar disorder was obtained by summing twice the value of the gait rating (weighted double because of the prominence of this sign in ACD) with the ratings of the two tests of coordinated movements of the lower limbs just mentioned.

Evaluation of dysarthria consisted of an oral motor assessment and perceptual speech analysis. A speech pathologist (K.J. K.) analyzed and rated the severity of the deviant speech dimensions during the examination and from audiotaped samples of spontaneous speech, expository speech, oral reading of the "Grandfather Passage" [38], diadochokinetic rates, duration of sustaining the vowels "ah" and "ee," and counting from 1 to 75 . We used the deviant speech dimensions defined by Darley and associates [38]. A quan- 
Table 1. Data on Patients in Group $A^{\mathrm{a}}$

\begin{tabular}{|c|c|c|c|c|c|c|c|c|c|c|c|c|c|}
\hline Patient & $\begin{array}{l}\text { Age } \\
\text { (yr) }\end{array}$ & Sex & $\begin{array}{l}\text { Duration of } \\
\text { Alcoholism }^{\mathrm{b}}\end{array}$ & $\begin{array}{l}\text { History of } \\
\text { Malnutrition }\end{array}$ & Nystagmus & Speech & Gait & $\mathrm{FNF}^{\mathrm{d}}$ & $\begin{array}{l}\text { RAM } \\
\text { Arms }^{e}\end{array}$ & $\mathrm{HKS}^{\mathrm{f}}$ & $\begin{array}{l}\text { RAM } \\
\text { Legs }^{8}\end{array}$ & Neuropathy ${ }^{\mathrm{h}}$ & Score \\
\hline 1 & 55 & $\mathbf{M}$ & 28 & Yes & + & + & +++ & 0 & + & ++ & ++ & + & 10 \\
\hline 2 & 54 & $\mathbf{M}$ & 38 & Yes & + & + & +++ & 0 & 0 & +++ & ++ & ++ & 11 \\
\hline 3 & 50 & $\mathrm{M}$ & 32 & Yes & + & + & ++ & + & + & +++ & ++ & ++ & 9 \\
\hline 4 & 55 & M & 35 & Yes & 0 & + & ++ & 0 & 0 & ++ & ++ & + & 8 \\
\hline 5 & 46 & M & 32 & Yes & + & + & ++ & 0 & 0 & ++ & ++ & $+t$ & 8 \\
\hline 6 & 42 & M & 24 & Yes & + & 0 & ++ & 0 & 0 & + & + & ++ & 6 \\
\hline 7 & 65 & M & 45 & Yes & 0 & 0 & + & 0 & 0 & ++ & + & ++ & 5 \\
\hline 8 & 57 & M & 27 & Yes & 0 & + & ++ & 0 & 0 & 0 & + & 0 & 5 \\
\hline 9 & 39 & $\mathbf{M}$ & 25 & Yes & + & 0 & + & 0 & 0 & + & + & ++ & 4 \\
\hline
\end{tabular}

${ }^{a}$ Characteristics of the 9 patients wich clinical neurological abnormalities consistent with the diagnosis of alcoholic cerebellar degeneration (Group $A$ ). The patients are listed in order with respect to the severity of the clinical signs, with the more severely affected cases at the beginning of the Table.

${ }^{b}$ The numbers indicate the years that each patient consumed alcohol irrespective of amount.

"A history of missing two or more meals per day over months or years.

dThe finger-nose-finger test of upper limb coordination.

e Rapidly alternating supination and pronation movements of the hands.

${ }^{f}$ The heel-knee-shin test of lower limb coordination.

sRapid tapping movements of the feet.

tEvidence of polyneuropathy such as decreased or absent ankle muscle stretch reflexes and diminished superficial sensation in the legs.

'An overall score was obtained to measure severity of the cerebellar vermal disorder. The score was obtained by summing twice the value of the gait rating, and adding to this the HKS rating and the RAM legs rating.

$0=$ none; $+=$ mild $;+=$ moderate; $+++=$ severe.

Table 2. Data on Patients in Group Ba

\begin{tabular}{|c|c|c|c|c|c|c|c|c|c|c|c|c|c|}
\hline Patient & $\begin{array}{l}\text { Age } \\
(\mathrm{yr})\end{array}$ & Sex & $\begin{array}{l}\text { Duration of } \\
\text { Alcoholism }\end{array}$ & $\begin{array}{l}\text { History of } \\
\text { Malnutrition }\end{array}$ & Nystagmus & Speech & Gait & FNF & $\begin{array}{l}\text { RAM } \\
\text { Arms }\end{array}$ & HKS & $\begin{array}{l}\text { RAM } \\
\text { Legs }\end{array}$ & Neuropathy & Score \\
\hline 10 & 55 & $\mathbf{M}$ & 39 & Yes & 0 & 0 & + & 0 & 0 & 0 & 0 & + & 0 \\
\hline 11 & 60 & $\mathrm{M}$ & 40 & No & 0 & 0 & + & 0 & 0 & 0 & 0 & + & 0 \\
\hline 12 & 51 & $\mathbf{M}$ & 34 & Yes & + & 0 & + & 0 & 0 & 0 & 0 & + & 0 \\
\hline 13 & 45 & M & 25 & Yes & 0 & 0 & 0 & 0 & 0 & 0 & 0 & 0 & 0 \\
\hline 14 & 51 & $\mathrm{M}$ & 30 & Yes & 0 & 0 & 0 & 0 & 0 & 0 & 0 & 0 & 0 \\
\hline
\end{tabular}

${ }^{3}$ Characteristics of the 5 patients with complaints of gait disorder but without signs of cerebellar dysfunction on clinical neurological examination (Group B). The gait disturbances observed were attributed to cervical spondylosis (Patient 12) or neuropathy (Patients 10, 11). See Table 1 for an explanation of the column heads.

titative rating scale extending from 0 (unaffected) to +++ (severely affected) was assigned to each deviant speech dimension as described previously [31].

Neuropsychological examinations were performed on each patient in a quiet, dedicated laboratory. The patients were judged to be cooperative and willing to engage in the procedures, and each examination was accomplished by an experienced technician who was naive as to the aims of the research project. All examinations were overseen by one of the neuropsychologist investigators (K. A. or S. B.). Each subject completed a battery of tasks that was chosen to reflect major areas of behavioral function known to be affected by changes in the CNS. These included general intellect, cognitive function such as learning and memory [39], psychomotor and sensory-perceptual behavior, and emotional and motivational aspects of behavior (Table 3 ).

Neuropsychological tests were scored in a standard manner and subsequently evaluated with $T$-score criteria $\{40]$. In the $T$-score evaluation, each subject was rated with regard to:
(1) level of any existing impairment; (2) lateralization of impairment, if any; (3) cognitive status; (4) perceptual status; (5) motor status; (6) specific impairment in frontal lobe functions; and (7) specific impairment in temporal lobe functions. The Halstead Impairment Index [41, 42] was calculated for each subject using the following subset of tests: Category Test, Tactual Performance Test, Speech Sounds Perception Test, Rhythm Test, and Finger 'Tapping (Dominant Hand). Data obtained with $T$-score criteria showed the same correlations as data obtained with the Halstead Impairment Index. Consequently, the data analysis presented in this paper is limited to the results of the Halstead Impairment Index.

Eight normal control subjects of similar age and sex to the patients were examined with PET. These subjects were recruited by local newspaper advertisements that had been reviewed and approved by the IRB of the UMMC prior to release. The control subjects had no history of neurological disease and no important abnormalities on neurological and general physical examination. Subjects who reported con- 
Table 3. Neuropsycbological Tests Administered

Wechsler Adult Intelligence Scale-Revised (WAIS-R)

Wide Range Achievement Test-Revised (WRAT-R)

Wechsler Memory Scale, Form I (with modification for measurement of delayed recall)

Lateral Dominance Examination

Sensory-Perceptual Examination

Aphasia Screening Test

Speech Sounds Perception Test

Rhythm Test

Category Test

Corsi Blocks

Controlled Oral Word Association (COWAT)

Buschke Selective Reminding

Finger Tapping

Grooved Pegboard

Grip Strength

Tactual Performance Test (TPT)

Trail Making Test (Forms A and B)

Minnesota Multiphasic Personality Inventory (MMPI)

suming more than four alcoholic drinks weekly were excluded from the control population. The subjects had no evidence of major psychopathological states as determined by interview and interpretation of formal testing by a neuropsychologist (K. A. or S. B.).

PET studies were conducted with the normal control subjects and patients with ACD lying supine, awake, and blindfolded in a quiet room. They were maintained under these conditions from 5 minutes before injection until completion of the scan. To ensure that similar levels and angles of PET scanning were obtained between patients, all patients were aligned parallel to the orbital meatal (OM) line using laser beams and the patients' heads were restrained by a band extending from the forehead onto the headholder. Scans were performed 30 to 75 minutes after intravenous injection of 5 to $10 \mathrm{mCi}$ of ${ }^{18} \mathrm{~F}$-FDG, which was synthesized with an adaptation of the method of Hamacher and colleagues [43].

In 11 of our patients with $A C D$ and the 8 normal control subjects, PET scans were performed with a TCC PCT $4600 \mathrm{~A}$ tomograph having an inplane resolution of $11-\mathrm{mm}$ full width at half maximum (FWHM) and a Z-axis resolution of $9.5-\mathrm{mm}$ FWHM. Five planes with $11.5-\mathrm{mm}$ center-tocenter separation were imaged simultaneously. Four sets of scans were taken per patient, including two interleaved sets through lower brain levels and two interleaved sets through higher brain levels for a total of 20 slices, each separated by $5.75 \mathrm{~mm}$. In 3 patients with $A C D$, PET scans were performed with a Siemens/CTI 931 08-12 scanner, which has a resolution of $5.5-\mathrm{mm}$ FWHM and a Z-axis resolution of 7.0$\mathrm{mm}$ FWHM. Fifteen planes with 6.75 -mm center-to-center separation were imaged simultaneously. Two sets of interleaved slices were taken per patient for a total of 30 slices, each separated by $3.75 \mathrm{~mm}$. The data sets from the 3 patients were smoothed with a three-dimensional filter to the resolution of the TCC scanner before region-of-interest (ROI) values were obtained, in order to minimize differences between scanners. Attenuation correction was calculated by fitting ellipses to the contour of the scalp outline and modified to account for attenuation from the headholder and skull.

Blood samples were collected from the radial artery for estimation of the arterial radioactive input function to brain. Local cerebral metabolic rate for glucose (ICMRglc) was calculated with a three-compartment model and a single-scan approximation [44] with gray-matter kinetic constants derived from normal subjects [45]. ROI data were acquired from the cerebellar hemispheres, cerebellar vermis, brainstem, thalamus, and cerebral cortex. PET images were viewed in the transverse, sagittal, and coronal planes. Data were collected from images obtained in the transverse plane by placing a $22 \times 11-\mathrm{mm}$ parallelogram over each cerebellar hemisphere, an $11 \times 19$-mm rectangle over the vermis, an $11 \times 15-\mathrm{mm}$ rectangle over the brainstem, and an $11 \times 11$ $\mathrm{mm}$ square over each thalamus [30] (Fig 1). Each ROI was centered over a local peak in ICMRglc. For reference, an individual image element [pixel] is $3.75 \times 3.75 \mathrm{~mm}$ in size. Data were obtained from two slices containing the cerebellum and brainstem and from one slice containing the thalamus. ROIs from the cerebellar vermis were posterior to the fourth ventricle. The brainstem ROI chiefly reflects the pons, but the mesencephalon or medulla oblongata could be partially represented. Data from the frontal regions were acquired by placing an elliptical region, $30 \times 22 \mathrm{~mm}$, over the rostral medial frontal area of the cerebral cortex, including both hemispheres (Table 4) (see Fig 1). Data were obtained from three consecutive slices beginning with the level containing the thalamus and moving upward. Data from the cerebral cortex were obtained by measuring ICMRglc in the cortical ribbon from six consecutive slices, beginning with the lowest slice containing the basal ganglia. This was accomplished with an algorithm that detects the outer edge of the cortical rim on an image that has been passed through a contrast-enhancing filter. The algorithm then identifies a band on the original image that extends inward from this edge until either the metabolic rate drops below the value on the outer edge of the rim or the band reaches a width of 15 $\mathrm{mm}$. An area weighted mean metabolic rate was computed for each of these ROIs. Normalized values were obtained by dividing individual ROI values by the mean value from the cerebral cortex over the six consecutive slices.

Analysis of ICMRglc in the inferomedial portion of the temporal lobes (hippocampus) was performed in the alcoholdependent patients and the normal control subjects. A $15 \times$ $19-\mathrm{mm}$ rectangular region with the long axis oriented anteroposteriorally was drawn over the hippocampal regions of the temporal lobes, located by reference to an anatomical arlas. The center of this region was located $28 \pm 1.9 \mathrm{~mm}$ to each side of the anteroposterior midline and $7.5 \pm 1.9 \mathrm{~mm}$ posterior to the most posteroinferior portion of the thalamus. The metabolic rate from this region was derived as the mean of the regional metabolic rates from the three scan planes from 2.4 to $3.5 \mathrm{~cm}$ above the canthomeatal line.

CT scans of the patients were obtained with a Picker Synerview 1200 SX. A subjective rating scale based on the expected size of the subarachnoid spaces given the patient's age (see Table 4) was devised to assess the degree of atrophy in the frontal region of the cerebral cortex and the cerebellum (see Table 4). 


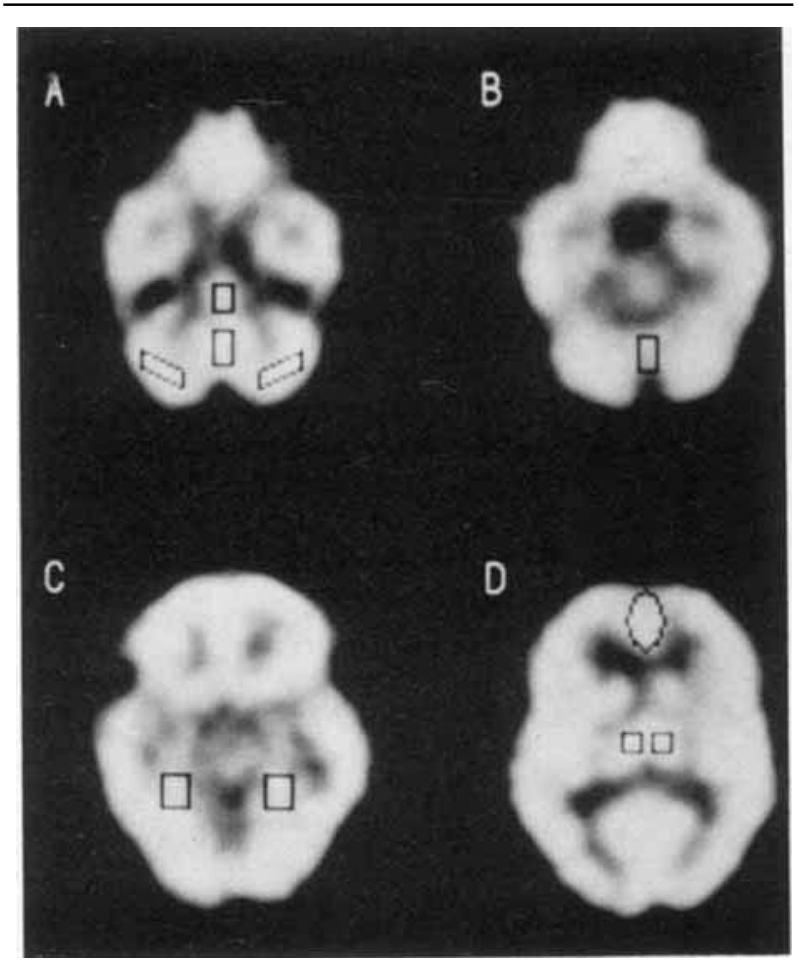

Fig 1. PET scans showing cerebral glucose utilization as medsured with ${ }^{18} \mathrm{~F}-2$-fluoro-2-deoxy-D-glucose. The scans are from a male patient (Patient 5) aged 46 years with alcobolic cerebellar degeneration. (A) Scan through the level of the cerebellar hemispheres and the base of the temporal and frontal lobes showing the locations of regions of interest (ROIs) in the cerebellar bemispberes, inferior cerebellar vermis, and brainstem. (B) Scan through the level of the superior cerebellar vermis and temporal and frontal lobes sbowing the location of the ROIs for the superior cerebellar vermis. (C) Scan tbrough the level of the temporal and frontal lobes showing the locations of the ROIs for the bippocampus. (D) Scan through the level of the frontal, temporal, parietal, and occipital lobes showing the locations of the ROIs for the medial frontal area of the cerebral cortex and the thalamus.

The neurological examinations and speech evaluations were graded by a neurologist (S. G.) and a speech pathologist (K. J. K.), respectively. The neuropsychological examinations were evaluated by a neuropsychologist (K. A. or S. B.) who was not involved directly in test administration. The abovementioned investigators were blinded to the results of the CT and PET studies. The PET studies were analyzed for ROIs by a physicist (R. A. K.) and a psychiatrist trained in imaging (J. G. M.), both of whom were blinded to the results of all the other studies. The CT scans were read by a neuroradiologist (J. A. B.) who was blinded to the other studies and was informed only of the patient's age. All data were shared among the investigators only after completion of measurements from all studies.

Student's $t$ tests were used for statistical analyses of the PET data to compare the alcohol-dependent patients with a group of age- and sex-matched normal control subjects. The Spearman rank correlation coefficient was used to assess the
Table 4. Rating Scale and Regions Scored on CT

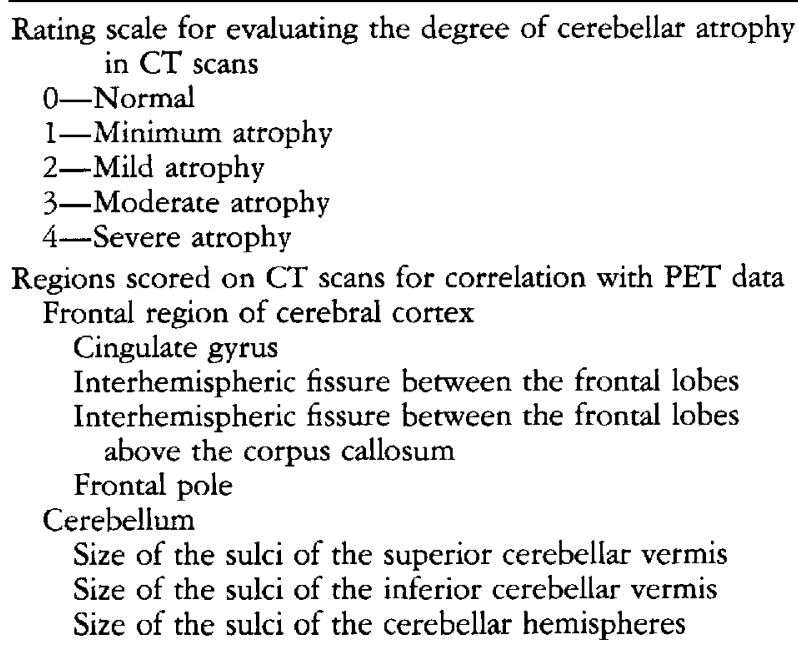

degree of relationship between $1 \mathrm{CMRglc}$, degree of atrophy as determined by CT, neurological function, and performance on neuropsychological tests.

\section{Results}

\section{Clinical Characteristics}

The patient group had an average age of 52 years and a range of 39 to 65 years (see Tables 1 and 2). All were severe chronic alcohol-dependent patients who had gradually increased their alcohol intake over the years preceding the neurological disorders. The average duration of alcohol intake was 32 years and the range was 24 to 45 years. All patients except one had a history of malnutrition, with food deprivation occurring frequently and, in many cases, lasting for days. The 1 patient without a history of malnutrition (Patient 11) had equivocal signs of cerebellar ataxia. None of the patients had a history or physical findings suggesting Wernicke-Korsakoff disease, and none had a family history of a cerebellar degeneration.

The patients complained chiefly about difficulty in standing and walking. Most also described incoordination of movements of the lower extremities, but none had noted speech difficulty or incoordination of upper limb movements. Neurological examination of most of these patients revealed unsteadiness of stance, ataxia of gait, and ataxia of lower extremity movements. These deficits ranged from mild to severe. Most patients had no abnormalities of extraocular movements except for nystagmus on lateral gaze. Most also had normal speech, though 6 patients had subtle to mild degrees of ataxic dysarthria. None had clinically detectable limb hypotonia. Patient 12 had a cervical myelopathy secondary to intervertebral disc disease with bilateral extensor plantar responses and weakness, spasticity, and hyperreflexia of all limbs. Most of the patients had a mild to moderate degree of peripheral neuropathy characterized by reduced or absent muscle stretch 
reflexes at the ankles and a stocking distribution of decreased superficial sensation in the legs, worse distally than proximally.

The patients were divided into two groups (A and B) based on the clinical neurological findings. Group A (see Table 1) consisted of 9 patients with clear clinical signs of $A C D$, including difficulty in standing with the legs together and ataxia of gait and leg movements. In Table 1 , the patients are ranked according to the severity of the neurological disorders. Group B (see Table 2) contained 5 chronic alcohol-dependent patients without clear signs of ACD. Patients 10 and 11 had no neurological abnormality on examination except for mild difficulty when walking in tandem, probably because of a peripheral neuropathy. As mentioned, Patient 12 had a cervical myelopathy with a gait disorder due to spasticity and mild weakness of the legs. Patients 13 and 14 had no neurological abnormalities on examination.

\section{PET Studies}

The PET scans of patients in Group A in comparison with those of the normal control subjects showed decreased glucose metabolic activity in the superior aspects of the cerebellar vermis (Fig 2). No abnormality was found in other portions of the cerebellum except in 1 patient who had hypometabolism of the cerebellar hemispheres as well as the vermis. Patients in Group B did not show decreased metabolic activity in the cerebellum. Patients in both Groups A and B, however, showed hypometabolism bilaterally in the medial frontal region of the cerebral cortex (see Fig 2). Typically, the hypometabolism extended in narrow bilateral medial bands extending anterior to posterior from the frontal poles to about the junction of the frontal and parietal lobes. This region corresponds approximately to the medial parts of both superior frontal and cingulate gyri.

Quantitative PET studies of 1CMRglc were normalized to mean whole brain cerebral cortex. In all 14 alcohol-dependent patients taken together (mean age, $52 \pm 8$ years), there were significant differences in the medial frontal area of the cerebral cortex when compared with normal control subjects (mean age, $52 \pm 7$ years) (Table 5). Studies of normalized lCMRglc in the 9 patients in Group A (mean age, $51 \pm 8$ years) demonstrated significant hypometabolism in the superior vermis and the rostral medial frontal area of the cerebral cortex when compared with normal control subjects (see Table 5). Studies of normalized lCMRglc in the 9 patients in Group A showed significant differences in the superior vermis but not the rostral medial frontal area of the cerebral cortex when compared with the 5 alcohol-dependent patients in Group B (mean age, $53 \pm 6$ years) (see Table 5). The increase of nor- malized ICMRglc in the superior cerebellar vermis in the 5 patients in Group B is not significantly different from the ICMRglc in the control group $(t=0.95$, $p>0.35$ ).

Studies of normalized ICMRglc were evaluated in relation to the severity of the neurological disorder as determined with the overall clinical score in all 14 alcohol-dependent patients (Table 6). Significant correlations were found between the severity of the neurological impairment and the degree of hypometabolism in the superior cerebellar vermis and the rostral medial frontal region of the cerebral cortex, but not in the other structures studied.

Studies of normalized ICMRglc were compared with the results of the neuropsychological studies summarized with the Halstead Impairment Index (Table 7). The results showed significant correlations between the Halstead Index and lCMRglc in the superior cerebellar vermis, but not in the other structures studied. The results of the Halstead Impairment Index were also positively associated with the overall clinical neurological score $\left(r_{s}=+0.77, p<0.005\right)$. Analysis of the individual tests comprising the Halstead Impairment Index in relation to normalized ICMRglc revealed a significant correlation between frontal lobe metabolism and Category Test Errors $\left(r_{\mathrm{s}}=-0.51, p<0.05\right)$.

Analysis of PET data obtained from the temporal lobes yielded no significant differences between the alcohol-dependent and control groups for left or right hippocampal absolute metabolic rates, whole-slice absolute metabolic rates, left to right hippocampal ratios, or left or right hippocampal to whole-slice ratios.

\section{CT Studies}

The CT scans revealed variable degrees of atrophy in the frontal, temporal, parietal, and occipital regions of the cerebral cortex, and also in the cerebellum. Atrophy in the brainstem could not be well quantified because of beam-hardening artifact. Since the PET studies revealed hypometabolism in the medial frontal area of the cerebral cortex of patients in Groups $A$ and $B$ and the superior cerebellar vermis of patients in Group A, we correlated lCMRglc in these regions with the degree of atrophy detected by CT. For Groups A and $\mathrm{B}$ together, a significant correlation was found between ICMRglc normalized to the cerebral cortex and CT atrophy for the rostral medial frontal area of the cerebral cortex bilaterally $\left(r_{\mathrm{s}}=-0.79, p<0.002\right)$, but not for the superior cerebellar vermis $\left(r_{\mathrm{s}}=\right.$ $-0.06)$, inferior cerebellar vermis $\left(r_{\mathrm{s}}=+0.33\right)$, or cerebellar hemispheres $\left(r_{s}=+0.12\right)$. For Group A alone, these values were significant for the rostral medial frontal area of the cerebral cortex $\left(r_{\mathrm{s}}=-0.75\right.$, $p<0.02$ ), but not for the superior cerebellar vermis, inferior cerebellar vermis, or cerebellar hemispheres. 


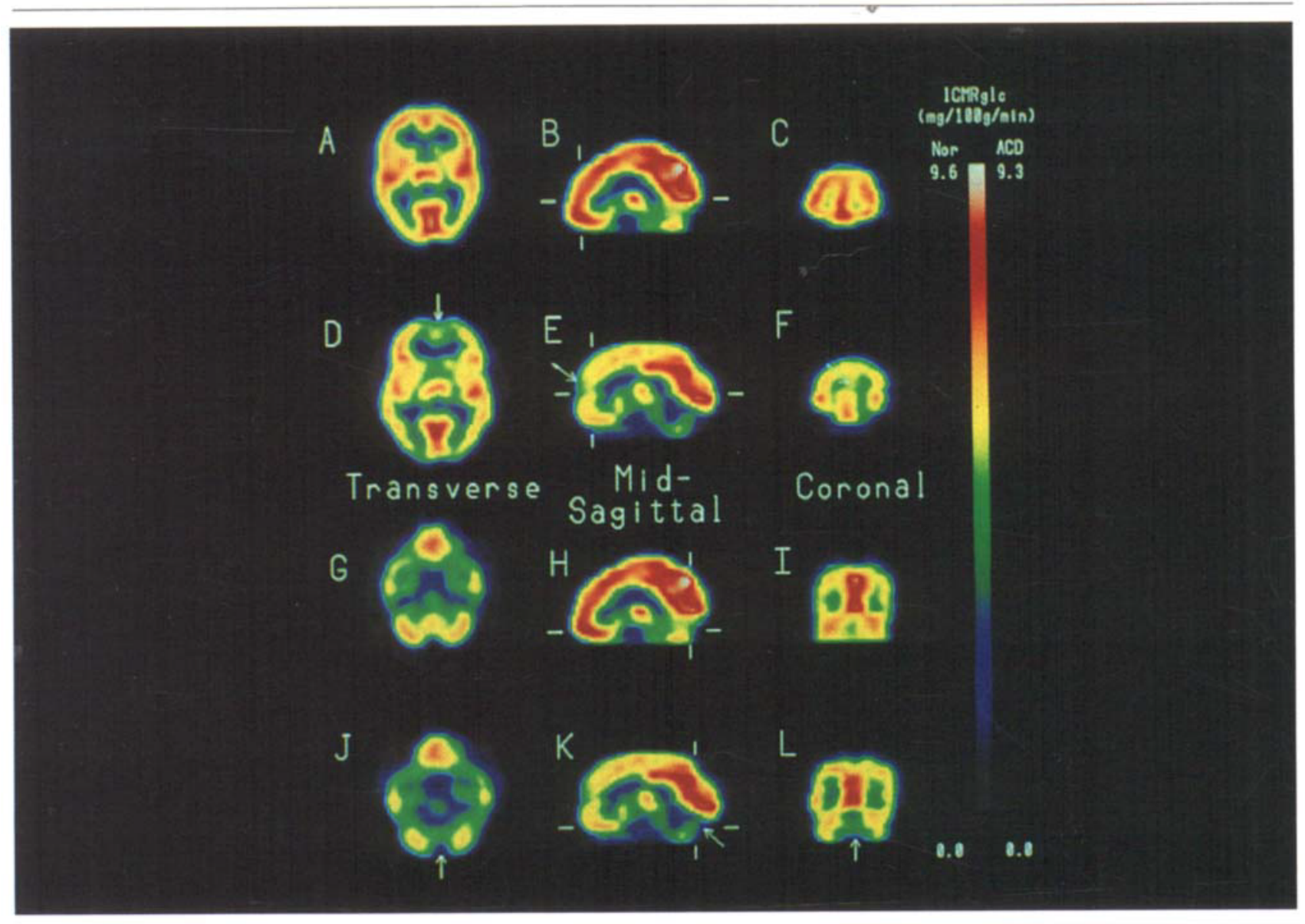

Fig 2. PET scans showing cerebral glucose utilization as measured with ${ }^{18} \mathrm{~F}-2-$ fluoro-2-deoxy-D-glucose. The scans are from a normal control male subject aged 57 years $(A, B, C, G, H$, and $I)$ and a male patient (Patient 5) aged 46 years with alcobolic cerebellar degeneration ( $A C D$ ) $(D, E, F J, K$, and $L)$. The scans show transverse sections at the level of the basal ganglia and thalamus ( $A$ and $D)$ and at the level of the cerebellum and the base of the temporal and frontal lobes ( $G$ and J); midsagittal sections tbrough the cerebral hemispheres and cerebellum $(B, E, H$, and $K$ ); and coronal sections tbrough the frontal lobes ( $C$ and $F$ ) and through the occipital lobes and cerebellum ( $I$ and $L)$. The short borizontal lines in the midsagittal sections $(B, E, H$, and $K)$ indicate the level of the corresponding transverse sections

$(A, D, G$, and J, respectively). The short vertical lines in the midsagittal sections $(B, E, H$, and $K)$ indicate the level of the corresponding coronal sections (C,F,I, and $L$, respectively). Note the bypometabolism in the medial frontal area of the cerebral cortex (arrows in $D, E$, and F) and in the anterior superior cerebellar vermis (arrows in J,K, and L). The color bar indicates local cerebral metabolic rates for glucose (lCMRglc) in $\mathrm{mg} / 100 \mathrm{gm} / \mathrm{min}$ in the normal control subject (range, 0.0 to 9.6) and the patient with ACD (range, 0.0 to 9.3). The left side of the brain corresponds to the left side of each image.
For Group B alone, the correlation for the rostral medial frontal area of the cerebral cortex was $r_{\mathrm{s}}=-0.70$, but did not reach significance at $p<0.05$ because of the small size of the group. The correlations were substantially lower for the superior cerebellar vermis, inferior cerebellar vermis, and cerebellar hemispheres. Comparison of neurological rating with the degree of atrophy in $\mathrm{CT}$ revealed no significant correlation for the rostral medial frontal area of the cerebral cortex $\left(r_{\mathrm{s}}=+0.22\right)$, superior cerebellar vermis $\left(r_{\mathrm{s}}=+0.26\right)$, inferior cerebellar vermis $\left(r_{\mathrm{s}}=+0.03\right)$, or cerebellar hemispheres $\left(r_{s}=+0.26\right)$. Comparison of the Halstead Impairment Index with the degree of atrophy on CT also revealed no significant correlation for the rostral medial frontal area of the cerebral cortex $\left(r_{\mathrm{s}}=\right.$ $+0.11)$, superior cerebellar vermis $\left(r_{s}=-0.07\right)$, inferior cerebellar vermis $\left(r_{\mathrm{s}}=+0.12\right)$, or cerebellar hemispheres $\left(r_{\mathrm{s}}=+0.30\right)$.

\section{Discussion}

The principal findings in this study are that patients with clinical evidence of ACD (Group A) showed hypometabolism in the anterior superior portions of the cerebellar vermis and in the medial frontal region 


\begin{tabular}{lllll}
\hline & & \multicolumn{3}{c}{ Alcoholic Patients } \\
\cline { 3 - 5 } Structure & $\begin{array}{l}\text { Control Subjects } \\
(\mathrm{n}=8)\end{array}$ & $\begin{array}{l}\text { Groups A and B } \\
(\mathrm{n}=14)\end{array}$ & $\begin{array}{l}\text { Group A } \\
(\mathrm{n}=9)\end{array}$ & $\begin{array}{l}\text { Group B } \\
\text { (n= 5) }\end{array}$ \\
\hline Superior cerebellar vermis & $0.94 \pm 0.12$ & $0.88 \pm 0.14$ & $0.82 \pm 0.12^{\mathrm{b}}$ & $1.00 \pm 0.05^{\mathrm{c}}$ \\
Inferior cerebellar vermis & $0.97 \pm 0.11$ & $0.95 \pm 0.10$ & $0.93 \pm 0.13$ & $0.97 \pm 0.04$ \\
Left cerebellar hemisphere & $1.07 \pm 0.16$ & $1.04 \pm 0.11$ & $1.04 \pm 0.12$ & $1.05 \pm 0.10$ \\
Right cerebellar hemisphere & $1.04 \pm 0.14$ & $1.04 \pm 0.11$ & $1.03 \pm 0.13$ & $1.06 \pm 0.10$ \\
Brainstem & $0.77 \pm 0.05$ & $0.81 \pm 0.10$ & $0.81 \pm 0.10$ & $0.82 \pm 0.11$ \\
Thalamus & $1.29 \pm 0.07$ & $1.22 \pm 0.12$ & $1.25 \pm 0.12$ & $1.16 \pm 0.09$ \\
Rostral medial frontal region of the cortex & $1.07 \pm 0.11$ & $0.94 \pm 0.06^{\mathrm{d}}$ & $0.91 \pm 0.05^{\mathrm{e}}$ & $0.98 \pm 0.07$ \\
\hline
\end{tabular}

${ }^{\mathrm{i}} \mathrm{Values}$ given are the mean $\pm \mathrm{SD}$.

${ }^{2} p<0.05$, Student's $t$ test (Group A patients versus control subjects).

$=p<0.01$, Student's $t$ test (Group A patients versus Group B patients).

${ }^{t} p<0.005$, Student's $t$ test (all alcoholic patients versus control subjects).

${ }^{\prime} p<0.005$, Student's $t$ test (Group A patients versus control subjects).

Table 6. Local Cerebral Metabolic Rates for Glucose Normalized to Whole Brain Cerebral Cortex in Normal Control Subjects Related to Degree of Neurological Impairment in All Alcobolic Patients ${ }^{2}$

\begin{tabular}{ll}
\hline Structure & $\begin{array}{l}r_{\mathrm{s}} \text { (Spearman Rank Order } \\
\text { Correlation Coefficient) }\end{array}$ \\
\hline Superior cerebellar vermis & $-0.73^{\mathrm{b}}$ \\
Inferior cerebellar vermis & -0.34 \\
Left cerebellar hemisphere & -0.26 \\
Right cerebellar hemisphere & -0.29 \\
Brainstem & -0.16 \\
Thalamus & +0.15 \\
Rostral medial frontal area & $-0.47^{\mathrm{c}}$ \\
\hline
\end{tabular}

aSeverity of neurological disorder graded with a single overall clinical neurological score (see Tables 1 and 2).

${ }^{\mathrm{b}} p<0.005$

$p<<0.05$.

of the cerebral cortex bilaterally. Chronic alcoholdependent patients without clinical evidence of ACD (Group B) did not have hypometabolism in the cerebellum, but this group did show hypometabolism in the medial frontal region of the cerebral cortex.

The location of the hypometabolism in the cerebellum of patients with ACD corresponds closely to the site of the major neuropathological changes in autopsy studies of ACD [2-4, 9]. The anterior and superior parts of the cerebellar vermis are involved neuropathologically, and in severe cases the pathological change extends into the hemispheral portions of the anterior lobes and the flocculi $[2,3]$. The cerebellar hemispheres are largely unaffected. The site shown to be hypometabolic in our study involves the superior portion of the cerebellar vermis, without significant changes in the inferior vermis or the hemispheres. The hypo-
Table 7. Local Cerebral Metabolic Rates for Glucose Normalized to Wbole Brain Cerebral Cortex in All Alcobolic Patients as a Function of the Halstead Impairment Index

\begin{tabular}{ll}
\hline Structure & $\begin{array}{l}r_{\mathrm{s}} \text { (Spearman Rank Order } \\
\text { Correlation Coefficient) }\end{array}$ \\
\hline Superior cerebellar vermis & $-0.58^{\mathrm{a}}$ \\
Inferior cerebellar vermis & -0.24 \\
Left cerebellar hemisphere & -0.27 \\
Right cerebellar hemisphere & -0.25 \\
Brainstem & -0.07 \\
Thalamus & +0.02 \\
Rostral medial frontal area & -0.31 \\
$\quad$ of cortex & \\
\hline
\end{tabular}

${ }^{a} p<0.05$.

metabolism observed in this site probably results from decreased cellular and synaptic activity because of neuronal degeneration. In ACD, essentially all cellular elements in the cerebellar cortex are known to be degenerated $[2,3,21]$; consequently, the cerebellar hypometabolism cannot be attributed to loss of any one single type of neuron.

The relationship of the degree of cerebellar hypometabolism to the severity of the clinical neurological disorder is significant, with greater degrees of hypometabolism associated with increasingly severe neurological impairments. Moreover, patients with a long history of severe alcohol dependence and malnutrition but without clinical neurological evidence of ACD did not show cerebellar hypometabolism.

The clinical neurological disorder resulting from $A C D$ consists of ataxia of gait and incoordination 
of leg movements without appreciable ataxia of arm movements, speech, or ocular movements [3]. Consequently, the clinical rating scale we devised involved evaluation only of gait and lower extremity movements; the gait disorder was weighted more than leg movement because of the importance of gait disorder to the diagnosis of ACD. Most of the alcohol-dependent patients without clinical signs of ACD had alcoholism and malnutrition (as evaluated by quantities of alcohol intake, patterns of alcohol intake, duration of alcohol dependence, and frequency and duration of nutritional deprivation) as severe as the patients who had signs of ACD. We anticipated that the group without clinical signs of ACD might show cerebellar hypometabolism, indicating that they have subclinical levels of ACD that may reach the clinical level with continued alcohol abuse and malnutrition. Since this was not found, we conclude that cerebellar hypometabolism is seen only in patients with clinical evidence of $A C D$ and not in those who are asymptomatic.

Anatomical imaging studies with CT or MR scans in chronic alcohol-dependent patients may show cerebellar atrophy, often in the anterior superior cerebellar vermis $[25,46,47]$, but also in the inferior vermis and hemispheres [23, 25, 26]. Many alcohol-dependent parients with cerebellar cortical atrophy demonstrated on CT do not show clinical signs of cerebellar degeneration [23], which is in keeping with the frequent observation of asymptomatic cerebellar degeneration in alcohol-dependent patients at autopsy [22]. In the present study, the degree of cerebellar degeneration on CT did not correlate well either with clinical neurological disorder or with the findings on PET study. This finding may result in part from problems in imaging the posterior fossa with $\mathrm{CT}$.

In contrast to the findings in the cerebellum, hypometabolism was found in the medial frontal area of the cerebral cortex bilaterally in the entire group of alcohol-dependent patients, whether cerebellar degeneration was present or not. The focus of the hypometabolism was in a medial strip of cortex in the frontal lobes, corresponding approximately to the medial parts of the superior frontal gyrus and the cingulate gyrus. Samson and associates [33], using ${ }^{18}$ F-FDG and PET in a study of 6 neurologically unaffected chronic alcohol-dependent patients, found no change in lCMRglc in cortical, subcortical, or cerebellar ROIs. Using a metabolic regional distribution index that reflects the distribution pattern of glucose utilization, however, they found evidence of selective medial frontal hypometabolism. The region found to be abnormal in their study appears to correspond approximately to the region found to be hypometabolic in our study. Full comparison of the areas affected in the two studies is difficult since the images in the study by Sansom and associates [33] were described only in the transverse plane. By viewing images reformatted into the sagittal plane, we found that the hypometabolic region extends as far posteriorly as the junction of the frontal and parietal lobes.

Anatomical imaging studies have demonstrated that generalized cerebral cortical atrophy occurs in individuals with chronic alcohol dependence $\{24,46,48-50\}$ and that the atrophy is partially reversible with abstinence [50]. In our study, the strong correlation between the degree of CT-determined atrophy in the rostral medial frontal region of the cerebral cortex and the observed decrease in lCMRglc at this site raises the possibility that the hypometabolism may, either in part or entirely, reflect loss of tissue. Anatomical studies have shown loss of neurons in the frontal part of the cerebral cortex from chronically alcohol-dependent patients [51]. From our data, we cannot determine whether the residual tissue in the frontal lobe is hypometabolic or is normally active metabolically. The absence of a significant correlation between the lCMRglc and the degree of atrophy in the cerebellar vermis would suggest that persisting cerebellar tissue is hypometabolic. Any conclusions concerning the effects of atrophy on metabolic rate in these studies must be tempered by limitations in the degree of resolution of our PET camera and the resolving power of CT scans, particularly with respect to structures in the posterior fossa.

Although hippocampal neuronal degeneration has been reported in alcohol-dependent patients $[3,34-$ 36], we did not find differences in 1CMRgic in the hippocampal regions between alcohol-dependent patients and normal control subjects, or even trends in this direction. Due to the limitations of image resolution for this relatively thin region of the cortex, however, we cannot rule out the possibility that a significant difference might be found in studies with greater image resolution.

The motor tasks in the neuropsychological tests corroborated the clinical neurological evaluations, showing a strong correlation with $1 \mathrm{CMRglc}$ in the superior vermis of the cerebellum. While the neuropsychological test results as a whole did not correlate with ICMRglc in the rostral medial frontal region of the cerebral cortex, they did indicate greater than normal difficulty in complex problem solving and other tasks traditionally associated with frontal lobe dysfunction [52]. One subtest, the categories task, requires complex problem solving in a context of shifting stimulus demand and is similar to other tasks that have been used to demonstrate frontal cortical function [53]. The abnormalities in categories task performance in the present study are positively correlated with the decline of ICMRglc in the medial frontal area of the cerebral cortex, and this correlation is independent of the cerebellar degeneration. The intriguing findings of medial 
frontal hypometabolism and the corresponding neuropsychological impairments require further study to determine whether they occur prior to severe chronic alcoholism [54] and may predispose to this condition, or whether these findings are the neuronal toxic result of chronic alcohol dependence.

Supported in part by National Institutes of Health grants NS 15655 and AA 07378.

We are indebted to the staff of the Division of Nuclear Medicine and of the Veterans Administration Medical Center for assistance with this srudy.

\section{References}

1. Romano J, Michael M Jr, Merritt $\mathbf{H H}$. Alcoholic cerebellar degeneration. Arch Neurol Psychiatry 1940;44:1230-1236

2. Victor M, Adams RD, Mancall EL. A restricted form of cerebellar degeneration occurring in alcoholic patients. Arch Neurol 1959;1:577-688

3. Victor M, Adams RD, Collins GH. The Wernicke-Korsakoff syndrome and related neurologic disorders due to alcoholism and malnutrition. 2nd ed. Philadelphia: Davis, 1989

4. Adams RD. Nutritional cerebellar degeneration. In: Vinken PJ, Bruyn GW, eds. Handbook of clinical neurology, vol 28. Amsterdam: North-Holland, 1976:271-283

5. Allsop J, Turner B. Cerebellar degeneration associated with chronic alcoholism. J Neurol Sci 1966:3:238-258

6. Costin JA, Smith JL, Emery S, Tomsak RL. Alcoholic downbeat nystagmus. Ann Ophthalmol 1980;12:1127-1131

7. Zasorin NL, Baloh RW. Downbeat nystagmus with alcoholic

8. Charness ME, Simon RP, Greenberg DA. Ethanol and the nervous system. N Engl J Med 1989;321:442-454

9. Victor M, Ferrendelli JA. The nutritional and metabolic diseases of the cerebellum. Clinical and pathological aspects. In: Fields WS, Willis WD, eds. The cerebellum in health and disease. St. Louis: Green, 1970:412-449

10. Diener HC, Dichgans J, Bacher M, Guschlbauer B. Improvement of ataxia in alcoholic cerebellar atrophy through alcohol abstinence. J Neurol 1984;231:258-262

11. Estrin WJ. Alcoholic cerebellar degeneration is not a dosedependent phenomenon. Alcoholism: Clin Exp Res 1987;11: 372-375

12. Mancall EL, McEntee WJ. Alterations of the cerebellar cortex in nutritional encephalopathy. Neurology 1965;15:303-313

13. Travares MA, Paula-Barbosa MM, Gray EG. A morphometric Golgi analysis of the Purkinje cell dendritic tree after long-term alcohol consumption in the adult rat. J Neurocytol 1983;12: 939-948

14. Chodoff $\mathrm{P}$, Auth T, Toupin H. Parenchymatous cortical cerebellar atrophy. J Nerv Ment Dis 1956;123:376-381

15. Decker JB, Wells CE, McDowell F. Cerebellar dysfunction associated with chronic alcoholism. Neurology 1959;9:361-366

16. Deitrich RA. Plasmalogen content of cerebellum samples from alcoholic and non-alcoholic humans. Alcoholism 1977;1:57-59

17. Kiessling $\mathrm{KH}$. The occurrence of acetaldehyde in various parts of rat brain after alcohol injection and its effect on pyruvate oxidation. Exp Cell Res 1962;27:367-368

18. Phillips SC. Qualitative and quantitative changes of mouse cerebellar synapses after chronic alcohol consumption and withdrawal. Exp Neurol 1985;88:748-756

19. Truitt EB Jr, Walsh MG. The role of acetaldehyde in the actions of alcohol. In: Kissin B, Begleiter H, eds. The biology of alcoholism, vol 1. New York: Plenum Press, 1971:161-195.
20. Kleinschmidt-DeMasters BK, Norenberg MD. Cerebellar de generation in the rat following rapid correction of hypona tremia. Ann Neurol 1981;10:561-565

21. Phillips SC, Harper CG, Kril J. A quantitative histological study of the cerebellar vermis in alcoholic patients. Brain 1987; 110:301-314

22. Torvik A, Torp S, Lindboe CF. Atrophy of the cerebellar vermis in ageing. A morphometric and histologic study. J Neurol Sci 1986;76:283-294

23. Hillbom M, Muuronen A, Holm L, Hindmarsh T. The clinical versus radiological diagnosis of alcoholic cerebellar degeneration. J Neurol Sci 1986;73:45-53

24. Dano P, Le Guyader J. Atrophie cerebrale et alcoolisme chro nique. Rev Neurol (Paris) 1988;144(3):202-208

25. Haubek A, Lee $\mathrm{K}$. Computed tomography in alcoholic cerebellar atrophy. Neuroradiology 1979;18:77-79

26. Koller WC, Glatt SL, Perlik S, et al. Cerebellar atrophy demon strated by computed tomography. Neurology 1981;31:405412

27. Melgaard B, Ahlgren P. Ataxia and cerebellar atrophy in chronic alcoholics. J Neurol 1986;233:13-15

28. Gilman S, Bloedel JR, Lechtenberg R. Disorders of the cerebellum. Philadelphia: Davis, 1981

29. Gilman S. Inherited ataxia. In: Johnson RT, ed. Current therapy in neurologic disease, vol 2. Toronto: Decker, 1987:224-232

30. Gilman S, Markel DS, Koeppe RA, et al. Cerebellar and brainstem hypometabolism in olivopontocerebellar atrophy detected with positron emission tomography. Ann Neurol 1988;23:223230

31. Kluin KJ, Gilman S, Markel DS, et al. Speech disorders in olivopontocerebellar atrophy correlate with positron emission tomography findings. Ann Neurol 1988;23:547-554

32. Rosenthal G, Gilman S, Koeppe RA, et al. Motor dysfunction rate studied with positron emission tomography. Ann Neurol $1988 ; 24: 414-419$

33. Samson $\mathrm{Y}$, Baron J-C, Feline A, et al. Local cerebral glucose utilisation in chronic alcoholics: a positron tomography study. J Neurol Neurosurg Psychiatry 1986;49:1165-1170

34. Freund G. Chronic central nervous system toxicity of alcohol. Annu Rev Pharmacol 1973;13:217-227

35. Butters N, Cermak L. Some analyses of amnesic syndromes in brain-damaged patients. In: Isaacson RI, Pribram KH, eds. The hippocampus: neurophysiology and behavior, vol 2. New York: Plenum Press, 1975:377-409

36. Walker DW, Barnes DE, Zornetzer SF, et al. Neuronal loss in hippocampus induced by prolonged ethanol consumption in rats. Science 1980;209:711-713

37. Gilman S, Adams K, Koeppe RA, Berent S. Cerebellar hypometabolism in alcoholic cerebellar degeneration studied with FDG and PET. Neurology 1988;38:365

38. Darley FL, Aronson AE, Brown JR. Motor speech disorders. Philadelphia: WB Saunders, 1975

39. Russell E. A multiple scoring method for the assessment of complex memory functions. J Consult Clin Psychol 1975;43: 800-809

40. Heaton RK, Grant I, Matthews CG. Differences in neuropsychological test performance associated with age, education, and sex. In: Grant I, Adams KM, eds. Neuropsychological assessment of neuropsychiatric disorders. New York: Oxford University Press, 1986

41. Halstead WC. Brain and intelligence: a quantitative study of the frontal lobes. Chicago: University of Chicago Press, 1947

42. Reitan RM, Davison LA, eds. Clinical neuropsychology: current status and applications. Washington, DC: VH Winston, 1974

43. Hamacher K, Coenen $\mathrm{HH}$, Stocklin G. Efficient stereospecific synthesis of NCA 2-[18 F]-fluoro-2-deoxy-D-glucose using ami- 
nopolyether supported direct nucleophilic substiturion. J Nucl Med 1986;27:235-238

44. Phelps ME, Huang SC, Hoffman EJ, et al. Tomographic measurement of local cerebral glucose metabolic rate in humans with (F-18) 2-fluoro-2-deoxy-D-glucose: validation of method. Ann Neurol 1979;6:371-388

45. Hawkins RA, Mazziotta JC, Phelps ME, et al. Cerebral glucose metabolism as a function of age in man: influence of the rate constants in the fluorodeoxyglucose method. J Cereb Blood Flow Metab 1983;3:250-253

46. Ramos A, Quintana F, Díez C, et al. CT findings in spinocerebellar degeneration. AJNR 1987:8:635-640

47. Diener HC, Muller A, Thron A, et al. Correlation of clinical signs with CT findings in patients with cerebellar disease. J Neurol 1986;233:5-12

48. Carlen PL, Wortzman G, Holgate RC, et al. Reversible cerebral atrophy in recently abstinent chronic alcoholics measured by computed tomography scans. Science 1978;200:1076-1078
49. Carlen PL, Wilkinson DA, Wortzman G, et al. Cerebral atrophy and functional deficits in alcoholics without clinically apparent liver disease. Neurology 1981;31:377-385

50. Carlen PL, Penn RD, Fornazzari L, et al. Computerized tomographic scan assessment of alcoholic brain damage and its potential reversibility. Alcoholism: Clin Exp Res 1986;10:226232

51. Harper C, Kril J. Patterns of neuronal loss in the cerebral cortex in chronic alcoholic patients. J Neurol Sci 1989;92:81-89

52. Parsons OA, Farr SD. The neuropsychology of drug and alcohol abuse. In: Filskov SB, Boll TJ, eds. The handbook of clinical neuropsychology. New York: John Wiley, 1981:320-365

53. Brody BA, Pribram KH. The role of frontal and parietal cortex in cognitive processing: tests of spatial and sequence functions. Brain 1978;101:607-633

54. Adams KM, Grant I. Influence of premorbid risk factors on neuropsychological performance in alcoholics. I Clin Exp Neuropsychol 1986;8:362-370 